\title{
Risk factor analysis of locoregional recurrence after sublobar resection in patients with clinical stage IA non-small cell lung cancer
}

\author{
Terumoto Koike, MD, PhD, ${ }^{\text {a,b }}$ Teruaki Koike, MD, PhD, ${ }^{\text {a }}$ Katsuo Yoshiya, MD, PhD, ${ }^{\text {a }}$ \\ Masanori Tsuchida, $\mathrm{MD}, \mathrm{PhD},{ }^{\mathrm{b}}$ and Shin-ichi Toyabe, $\mathrm{MD}, \mathrm{PhD}^{\mathrm{c}}$
}

\begin{abstract}
Objective: Although lobectomy is the standard surgical procedure for operable non-small cell lung cancer (NSCLC), sublobar resection also has been undertaken for various reasons. The aim of this study was to identify risk factors of locoregional recurrence and poor disease-specific survival in patients with clinical stage IA NSCLC undergoing sublobar resection.
\end{abstract}

\begin{abstract}
Methods: We retrospectively reviewed 328 patients with clinical stage IA NSCLC who underwent segmentectomy or wedge resection. Demographic, clinical, and pathologic factors were analyzed using the log-rank test as univariate analyses, and all factors were entered into a Cox proportional hazards regression model for multivariate analyses to identify independent predictors of locoregional recurrence and poor disease-specific survival.
\end{abstract}

Results: The 5- and 10-year locoregional recurrence-free probabilities were $84.8 \%$ and $83.6 \%$, respectively, and the 5- and 10-year disease-specific survivals were $83.6 \%$ and $73.6 \%$, respectively. Four independent predictors of locoregional recurrence were identified: wedge resection (hazard ratio [HR], 5.787), microscopic positive surgical margin (HR, 3.888), visceral pleural invasion (HR, 2.272), and lymphatic permeation (HR, 3.824). Independent predictors of poor disease-specific survival were identified as follows: smoking status (Brinkman Index; HR, 1.001), wedge resection (HR, 3.183), microscopic positive surgical margin (HR, 3.211), visceral pleural invasion (HR, 2.553), and lymphatic permeation (HR, 3.223). All 4 predictors of locoregional recurrence also were identified as independent predictors of poor disease-specific survival.

Conclusions: Segmentectomy should be the surgical procedure of first choice in patients with clinical stage IA NSCLC who are being considered for sublobar resection. Patients having tumors presenting with no suspicious of pleural involvement would be suitable candidates for sublobar resection. (J Thorac Cardiovasc Surg 2013;146:372-8)

Earn CME credits at

http://cme.ctsnetjournals.org

Ever since the Lung Cancer Study Group (LCSG) performed a prospective and randomized study of limited resection versus lobectomy in patients with peripheral T1 lung cancers and reported the results of the study in $1995,{ }^{1}$ lobectomy has been the standard surgical procedure for early-stage non-small cell lung cancer (NSCLC). The LCSG reported an approximately 3 -fold increase of the locoregional recurrence rate in patients who underwent limited resection compared with that in patients who underwent lobectomy.

From the Division of Chest Surgery, ${ }^{a}$ Niigata Cancer Center Hospital, Niigata, Japan; Division of Thoracic and Cardiovascular Surgery, ${ }^{\mathrm{b}}$ Niigata University Graduate School of Medical and Dental Sciences, Niigata, Japan; and Niigata University Crisis Management Office, ${ }^{\mathrm{c}}$ Niigata University Hospital, Niigata, Japan.

Disclosures: Authors have nothing to disclose with regard to commercial support.

Received for publication Aug 21, 2012; revisions received Jan 16, 2013; accepted for publication Feb 27, 2013.

Address for reprints: Teruaki Koike, MD, PhD, Division of Chest Surgery, Niigata Cancer Center Hospital, 2-15-3 Kawagishi-cho, Chuo-ku, Niigata, Niigata, Japan 951-8566 (E-mail: koike@niigata-cc.jp).

$0022-5223 / \$ 36.00$

Copyright (c) 2013 by The American Association for Thoracic Surgery

http://dx.doi.org/10.1016/j.jtcvs.2013.02.057
However, sublobar resection, such as segmentectomy and wedge resection, could benefit patients with NSCLC with comorbid disease or decreased pulmonary function, ${ }^{2}$ or older patients with a limited life expectancy. ${ }^{3}$ Moreover, several prospective trials of intentional sublobar resection for small peripheral NSCLC in patients with adequate pulmonary function for lobectomy have been conducted recently, ${ }^{4,5}$ on the basis of the evidence that smaller lesions are likely to represent an earlier stage and potentially more liable to cure. ${ }^{6}$ Furthermore, favorable outcomes of sublobar resection have been reported in patients with NSCLC with a relatively high percent area of ground-glass opacity on computed tomography $(\mathrm{CT}),{ }^{7,8}$ which is considered to be indicative of less invasive adenocarcinoma. Despite the reported equivalent survival between patients undergoing intentional sublobar resection and lobectomy, a suitable population for sublobar resection with a low risk of locoregional recurrence has still not been established.

In the current study, we retrospectively reviewed the data of patients with clinical stage IA NSCLC who underwent segmentectomy or wedge resection at the Niigata Cancer Center Hospital in an attempt to identify the independent demographic, clinical, and pathologic predictors of locoregional recurrence and poor disease-specific survival. 


\section{Abbreviations and Acronyms \\ $\mathrm{BI}=$ Brinkman Index \\ CEA $\quad=$ carcinoembryonic antigen \\ $\mathrm{CI}=$ confidence interval \\ Cons/Tumor ratio $=$ consolidation/tumor ratio \\ $\mathrm{CT}=$ computed tomography \\ $\mathrm{HR} \quad=$ hazard ratio \\ LCSG $\quad=$ Lung Cancer Study Group \\ NSCLC = non-small cell lung cancer \\ PET = positron emission tomography}

\section{MATERIALS AND METHODS \\ Patients}

This study was approved by the institutional review board of the Niigata Cancer Center Hospital. Because this was a retrospective study, the need to obtain written informed consent from each patient was waived.

In this study, we conducted a retrospective evaluation of patients with clinical stage IA NSCLC, according to the 7th edition of the TNM classification, ${ }^{9}$ who had undergone segmentectomy or wedge resection at the Niigata Cancer Center Hospital, Niigata, Japan. Patients meeting the following criteria were excluded: with a nonperipherally located carcinoma visualized by bronchoscopy, evidence of multiple lung carcinomas, evidence of pleural dissemination, positive pleural effusion or lavage cytology, and planned adjuvant therapy after surgical resection (Figure 1). Patients with pathologically diagnosed adenocarcinoma in situ also were excluded, because this is a preinvasive state of adenocarcinoma, and locoregional recurrence is not expected to develop even after sublobar resection. ${ }^{7,8}$

A total of 328 patients with clinical stage IA NSCLC treated between January 1998 and December 2009 fulfilled the inclusion criteria for this study and were enrolled. Of the eligible patients, 196 were male and 132 were female, and the median age was 71 years (range, 42-89 years; Table 1). All the patients had clinical stage IA disease, and 80 of the 328 patients $(24.4 \%)$ had clinical T1b disease. Segmentectomy was performed in 216 patients $(65.9 \%)$, and wedge resection was performed in 112 patients $(34.1 \%)$; systematic mediastinal lymphadenectomy was performed in 86 patients $(26.2 \%)$. Tumor histology was adenocarcinoma in 269 patients $(82.0 \%)$, squamous cell carcinoma in 46 patients $(14.0 \%)$, and other cell types in 13 patients $(4.0 \%)$.

\section{Preoperative Evaluation}

All patients underwent preoperative staging with $\mathrm{CT}$ and pulmonary function testing. Additional diagnostic testing (brain magnetic resonance imaging and bone scintigraphy) was performed at the discretion of the individual physician, according to the patient's symptoms and clinical findings. Because positron emission tomography (PET) combined with CT was introduced at the Niigata Cancer Center Hospital in 2010, PET-CT was not used for preoperative staging in any of the patients enrolled in this study. No invasive modalities for mediastinal lymph node staging, such as mediastinoscopy or endobronchial ultrasound-guided transbronchial needle aspiration, were used preoperatively in any of these patients.

\section{Surgery}

Sublobar resection is selected for the treatment of high-risk subgroup of patients with decreased pulmonary function or comorbid disease, such as previous treatment for other malignancies, underlying pulmonary disease, underlying heart disease, and advanced age. In patients with tumors presenting with a low consolidation/tumor (Cons/Tumor) ratio on CT (a maximum diameter of consolidation divided by the maximum tumor diameter on CT) ${ }^{10}$ intentional segmentectomy or wedge resection was performed, and the extent of pulmonary resection was determined depending on the tumor size and location. Intentional segmentectomy also was performed in patients with cT1a with adequate pulmonary function. ${ }^{4}$ In these patients, intraoperative surgical margin lavage cytology was performed in patients in whom the tumor was close to the surgical margin, and negative cytology was confirmed. In some intentionally indicated patients, the N1 nodes were pathologically assessed intraoperatively, and if positive, the surgical procedure was converted to lobectomy. In patients at high risk for lobectomy or who underwent wedge resection, the intraoperative pathologic N1 node assessment was performed only when a positive N1 node was strongly suggested by intraoperative findings.

\section{Postoperative Follow-up}

According to the hospital's clinical routine of postoperative followup, postoperative patients were seen approximately every 3 months after sublobar resection, and the follow-up evaluation included physical examination, chest radiography, and blood examination including measurements of tumor markers. A CT scan of the thorax was performed every year for the first 5 years post-resection and then as necessary after the first 5 years post-resection. If the patient showed any symptoms or signs of recurrence, further evaluations were performed, including CT, brain magnetic resonance imaging, and bone scintigraphy. Since 2010, we have performed PET-CT when necessary. Recurrence was defined as locoregional if it occurred within the same lobe, or in the ipsilateral thoracic cavity, and hilum or mediastinal lymph nodes. Newly developed lung lesions only in the same lobe were scored as local recurrence unless the lesions showed different histologic subtypes from a primary lung carcinoma.

\section{Data}

We reviewed the medical records of each patient to obtain data, including age ( $\leq 71$ years or $>71$ years), gender, smoking status (Brinkman In$\operatorname{dex}[\mathrm{BI}],{ }^{11} 0,>0-600$, or $\left.>600\right)$, preoperative serum carcinoembryonic antigen (CEA) level ( $<5 \mathrm{ng} / \mathrm{mL}$ or $\geq 5 \mathrm{ng} / \mathrm{mL}$ ), tumor location (right upper or middle, right lower, left upper, or left lower lobe), tumor size on preoperative radiologic imaging ( $\leq 2.0$ or $2.1-3.0 \mathrm{~cm}$ ), Cons/Tumor ratio on $\mathrm{CT}^{10}(\leq 75 \%$ or $>75 \%)$, reason for sublobar resection (compromised or intentional), extent of pulmonary resection (segmentectomy or wedge resection), extent of lymphadenectomy (sampling only or systematic mediastinal lymphadenectomy), tumor histology (adenocarcinoma, squamous cell carcinoma, or other cell types), tumor size in the resected lung specimens $(\leq 2.0,2.1-3.0$, or $3.1-5.0 \mathrm{~cm})$, microscopic surgical margin (negative or positive), pathologically diagnosed lymph node metastasis (absent or present), visceral pleural invasion (absent or present), lymphatic permeation (absent or present), and vascular invasion (absent or present).

For the univariate analysis to determine the influence of age, the patients were divided into 2 groups by the median age of 71 years. The BI was scored as the number of cigarettes smoked per day multiplied by the number of years smoked. ${ }^{11}$ The cutoff point of the BI was set at 600 on the basis of a previous report, ${ }^{12}$ and a BI greater than 600 indicated heavy smoking. The cutoff for the normal upper limit of serum CEA was set at $5 \mathrm{ng} / \mathrm{mL}$. The Cons/Tumor ratio was defined as the maximum diameter of consolidation divided by the maximum tumor diameter on $\mathrm{CT}^{10}$ and evaluated with the reconstructed thin-section helical CT scan. The patients were divided into 2 groups by $75 \%$ of Cons/Tumor ratio as used in a previous report, ${ }^{13}$ and a high Cons/Tumor ratio indicated an aggressive disease in the report. Presence or absence of visceral pleural invasion, lymphatic permeation, and vascular invasion were evaluated by pathologists in specimens stained with hematoxylin-eosin and Elastica van Gieson stain. 


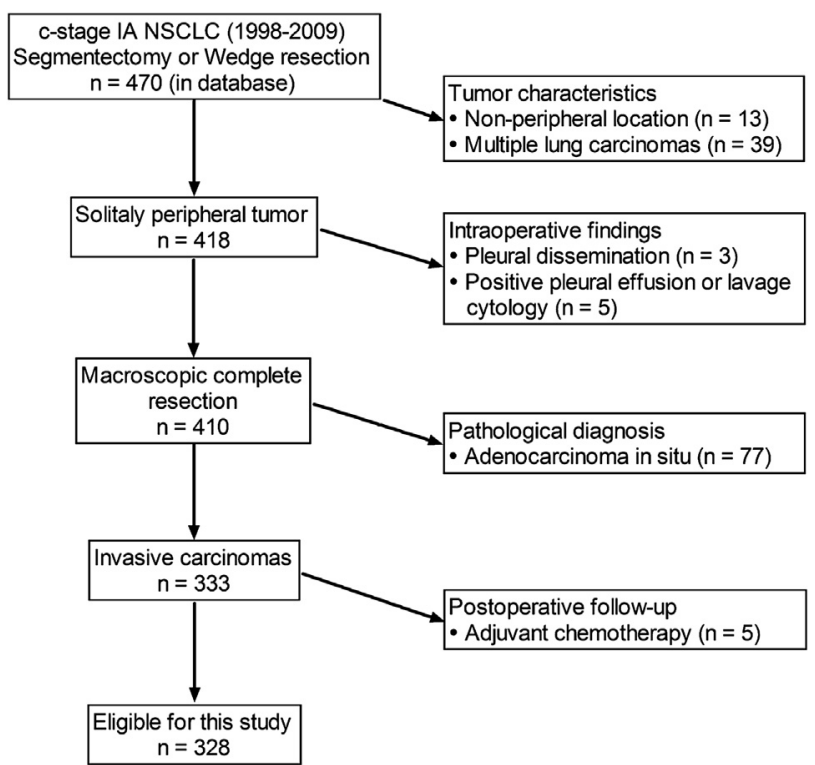

FIGURE 1. CONSORT-style diagram illustrating the selection of patients included in the data analysis. NSCLC, Non-small cell lung cancer.

\section{Statistical Analysis}

The locoregional recurrence-free probability was measured from the date of surgery to the date of detection of the first locoregional recurrence or the date of last follow-up. Locoregional recurrence was scored only when it was identified as the first recurrence site. The length of diseasespecific survival was estimated as the interval from the date of surgery to the date of a specific cause of death or the date of last follow-up. For the univariate analyses, the locoregional recurrence-free probability and disease-specific survival were calculated using the Kaplan-Meier method, and predictors of locoregional recurrence and poor disease-specific survival were analyzed with the log-rank test. The Bonferroni correction for multiple comparisons was used for each individual comparison. The dichotomized data were used only for the univariate analysis in each continuous variable (age, BI, preoperative serum CEA level, tumor size on preoperative radiologic imaging, Cons/Tumor ratio on CT, and tumor size in the resected lung specimens). All factors were entered into a Cox proportional regression hazard model for multivariate analysis. In the multivariate analysis, categoric variables (sex, tumor location, reason for sublobar resection, pulmonary resection extent, lymphadenectomy extent, tumor histology, microscopic surgical margin, lymph node metastasis, lymphatic permeation, and vascular invasion) and continuous variables (age [years], BI, preoperative serum CEA level [nanograms/milliliter], tumor size on preoperative radiologic imaging [centimeters], Cons/Tumor ratio on CT [percent], and tumor size in the resected lung specimens [centimeter]) were assessed to identify independent predictors. A stepwise selection method was used to identify the risk factors of locoregional recurrence and independent prognostic factors. The assumption of proportionality in each significant variable was verified using log-minus-log survival plots. All analyses were performed using IBM SPSS 20.0 (IBM Co, Chicago, Ill).

\section{RESULTS}

During the follow-up period, locoregional recurrence developed in 51 patients, represented by pulmonary metastasis in the same lobe in 34 cases, metastasis in the intrathoracic lymph nodes in 12 cases, pleural dissemination in 2 cases, and both pulmonary metastasis in the same lobe and metastasis in the intrathoracic lymph nodes in 3 cases. In the 15 patients in whom intrathoracic lymph node metastases developed, 3 underwent mediastinal lymphadenectomy (data not shown). The 5- and 10-year locoregional recurrence-free probabilities were $84.8 \%$ and $78.2 \%$, respectively, with a median follow-up period of 58 months (range, 2-162 months; Figure 2, $A$ ). The 5- and 10 -year disease-specific survivals were $83.6 \%$ and $73.6 \%$, respectively, with a median follow-up period of 62 months (range, 3-170 months; Figure 2, B).

The univariate analyses identified the following significant factors of a reduced locoregional recurrence-free probability: age greater than 71 years, male gender, BI greater than 600 , serum CEA $5 \mathrm{ng} / \mathrm{mL}$ or greater, radiologic tumor size 2.1 to $3.0 \mathrm{~cm}$, Cons/Tumor ratio on CT greater than $75 \%$, indication for compromised patients, wedge resection, lymph node sampling only, squamous cell carcinoma, microscopic positive surgical margin, lymph node metastasis, visceral pleural invasion, lymphatic permeation, and vascular invasion (Table 1). For disease-specific survival, the following were identified as significant factors in the univariate analyses: age greater than 71 years, male gender, BI greater than 600 , serum CEA $5 \mathrm{ng} / \mathrm{mL}$ or greater, radiologic tumor size 2.1 to $3.0 \mathrm{~cm}$, Cons/Tumor ratio on CT greater than $75 \%$, indication for compromised patients, wedge resection, squamous cell carcinoma, positive microscopic surgical margin, lymph node metastasis, visceral pleural invasion, lymphatic permeation, and vascular invasion (Table 1).

A multivariate analysis using the Cox proportional hazards regression model revealed the following 4 significant independent predictors of locoregional recurrence: wedge resection (hazard ratio [HR], 5.787; 95\% confidence interval $[\mathrm{CI}], 3.018-11.098, P<.001)$, microscopic positive surgical margin (HR, 3.888; 95\% CI, 1.634-9.255; $P=.002$ ), visceral pleural invasion (HR, 2.272; 95\% CI, 1.282-4.027; $P=.005$ ), and lymphatic permeation (HR, 3.824; $95 \% \mathrm{CI}$, 2.028-7.210; $P<.001$; Table 2 ). In addition, the following were identified as the factors associated with a poor disease-specific survival: smoking status (BI; HR, 1.001; 95\% CI, 1.000-1.001; $P=.003$ ), wedge resection (HR, 3.183; 95\% CI, 1.756-5.771; $P<.001$ ), microscopic positive surgical margin (HR, 3.211; 95\% CI, 1.4277.255; $P=.005$ ), visceral pleural invasion (HR, 2.553; $95 \% \mathrm{CI}, 1.503-4.388 ; P=.001)$, and lymphatic permeation (HR, 3.223; 95\% CI, 1.773-5.860; $P<.001$; Table 2). Among the 4 independent predictors of locoregional recurrence identified, all 4 factors also were identified as independent predictors of poor disease-specific survival.

\section{DISCUSSION}

In the present study, we retrospectively reviewed the data of 328 patients with clinical stage IA NSCLC who underwent sublobar resection in an attempt to identify 
TABLE 1. Demographic, clinical, and pathologic characteristics, and univariate analysis of locoregional recurrence-free probability and diseasespecific survival for 328 patients with clinical stage IA non-small cell lung cancer undergoing sublobar resection

\begin{tabular}{|c|c|c|c|c|c|}
\hline Variable & No. of patients $(\%)$ & $\begin{array}{c}\text { 5-y locoregional recurrence-free } \\
\text { probability }(\%)\end{array}$ & $\begin{array}{l}\text { Univariate } \\
P \text { value }\end{array}$ & $\begin{array}{l}\text { 5-y disease-specific } \\
\text { survival }(\%)\end{array}$ & $\begin{array}{c}\text { Univariate } \\
P \text { value }\end{array}$ \\
\hline All cases & $328(100)$ & 84.8 & & 83.6 & \\
\hline \multicolumn{6}{|l|}{ Age } \\
\hline$\leq 71 \mathrm{y}$ & $177(54)$ & 90.1 & & 89.8 & \\
\hline$>71 \mathrm{y}$ & $151(46)$ & 78.5 & .0019 & 76.6 & .0019 \\
\hline \multicolumn{6}{|l|}{ Sex } \\
\hline Male & $196(60)$ & 78.2 & & 77.6 & \\
\hline Female & $132(40)$ & 93.6 & .0019 & 88.6 & $.007 \Phi$ \\
\hline \multicolumn{6}{|l|}{ Smoking status (BI*) } \\
\hline 0 & $138(42)$ & 92.4 & & 92.0 & \\
\hline$>0-600$ & $33(10)$ & 93.4 & & 83.2 & \\
\hline$>600$ & $157(48) \S$ & 75.5 & .0029 & 76.1 & .0039 \\
\hline \multicolumn{6}{|l|}{$\mathrm{CEA} \dagger$} \\
\hline Within normal range & $277(84)$ & 88.6 & & 88.6 & \\
\hline Elevated & $51(16)$ & 63.1 & $<.001 \rrbracket$ & 57.1 & $<.0019$ \\
\hline \multicolumn{6}{|l|}{ Tumor location } \\
\hline Right upper or middle lobe & $113(35)$ & 86.2 & & 82.5 & \\
\hline Right lower lobe & $69(21)$ & 81.7 & & 82.2 & \\
\hline Left upper lobe & $89(27)$ & 90.1 & & 87.9 & \\
\hline Left lower lobe & $57(17)$ & 76.6 & .264 & 81.0 & .764 \\
\hline \multicolumn{6}{|l|}{ Tumor size, radiologic } \\
\hline$\leq 2.0 \mathrm{~cm}$ & $248(76)$ & 88.8 & & 88.3 & \\
\hline $2.1-3.0 \mathrm{~cm}$ & $80(24)$ & 72.1 & $<.0019$ & 69.1 & .0019 \\
\hline \multicolumn{6}{|l|}{ Cons/Tumor ratio $\ddagger$} \\
\hline$\leq 75 \%$ & $89(27)$ & 96.5 & & 97.5 & \\
\hline$>75 \%$ & $239(63)$ & 80.5 & $<.0019$ & 78.7 & $<.0019$ \\
\hline \multicolumn{6}{|l|}{ Reason for sublobar resection } \\
\hline Compromised & $160(49)$ & 74.8 & & 79.3 & \\
\hline Intentional & $168(51)$ & 93.5 & $<.0019$ & 93.0 & $<.0019$ \\
\hline \multicolumn{6}{|l|}{ Pulmonary resection extent } \\
\hline Segmentectomy & $216(66)$ & 93.7 & & 91.3 & \\
\hline Wedge resection & $112(34)$ & 66.0 & $<.0019$ & 68.0 & $<.0019$ \\
\hline \multicolumn{6}{|l|}{ Lymphadenectomy extent } \\
\hline Sampling only & $242(74)$ & 82.7 & & 81.4 & \\
\hline Systematic mediastinal node dissection & $86(26)$ & 91.0 & .0409 & 90.1 & .095 \\
\hline \multicolumn{6}{|l|}{ Tumor histology } \\
\hline Adenocarcinoma & $269(82)$ & 88.5 & & 86.6 & \\
\hline Squamous cell carcinoma & $46(14) \|$ & 62.6 & & 72.4 & \\
\hline Others & $13(4)$ & 61.5 & .0029 & 61.5 & .0079 \\
\hline \multicolumn{6}{|l|}{ Tumor size, pathologic } \\
\hline$\leq 2.0 \mathrm{~cm}$ & $246(75)$ & 86.9 & & 85.4 & \\
\hline $2.1-3.0 \mathrm{~cm}$ & $64(20)$ & 76.0 & & 73.5 & \\
\hline $3.1-5.0 \mathrm{~cm}$ & $18(5)$ & 87.2 & .131 & 94.4 & .112 \\
\hline \multicolumn{6}{|l|}{ Microscopic surgical margin } \\
\hline Positive & $11(3)$ & 24.2 & & 18.2 & \\
\hline Negative & 317 (97) & 86.9 & $<.0019$ & 85.9 & $<.0019$ \\
\hline \multicolumn{6}{|l|}{ Lymph node metastasis } \\
\hline Absent & $318(97)$ & 86.3 & & 84.4 & \\
\hline Present & $10(3)$ & 40.0 & $<.001 \uparrow$ & 60.0 & .0029 \\
\hline \multicolumn{6}{|l|}{ Visceral pleural invasion } \\
\hline Absent & $260(79)$ & 89.0 & & 88.9 & \\
\hline Present & $68(21)$ & 68.5 & $<.001 \Phi$ & 65.1 & $<.001 \Phi$ \\
\hline
\end{tabular}


TABLE 1. Continued

\begin{tabular}{|c|c|c|c|c|c|}
\hline Variable & No. of patients $(\%)$ & $\begin{array}{c}\text { 5-y locoregional recurrence-free } \\
\text { probability }(\%)\end{array}$ & $\begin{array}{l}\text { Univariate } \\
P \text { value }\end{array}$ & $\begin{array}{l}\text { 5-y disease-specific } \\
\text { survival }(\%)\end{array}$ & $\begin{array}{c}\text { Univariate } \\
P \text { value }\end{array}$ \\
\hline \multicolumn{6}{|l|}{ Lymphatic permeation } \\
\hline Absent & $278(85)$ & 88.2 & & 86.6 & \\
\hline Present & $50(15)$ & 65.4 & $<.001 \rrbracket$ & 66.7 & $<.001 \uparrow$ \\
\hline \multicolumn{6}{|l|}{ Vascular invasion } \\
\hline Absent & $258(79)$ & 86.3 & & 87.7 & \\
\hline Present & $70(21)$ & 71.1 & .003 व & 68.3 & $<.0019$ \\
\hline
\end{tabular}

$B I$, Brinkman Index; $C E A$, carcinoembryonic antigen. *Scored as the number of cigarettes smoked per day multiplied by the number of years since smoking started. $\dagger$ Preoperative serum CEA level, normal upper limit at $5 \mathrm{ng} / \mathrm{mL}$. $\ddagger$ Cons/Tumor ratio, consolidation/tumor ratio, a maximum diameter of consolidation divided by the maximum tumor diameter on CT. $\S$ Locoregional recurrence-free probability: 0 versus $\geq 600: P=.006$; disease-specific survival: 0 versus $\geq 600: P=.003$. $\|$ Locoregional recurrence-free probability: adenocarcinoma versus squamous cell carcinoma: $P=.021$; disease-specific survival: adenocarcinoma versus squamous cell carcinoma: $P=.027$. $\uparrow$ Indicates significance.

independent predictors of locoregional recurrence and poor disease-specific survival. The multivariate analyses identified wedge resection, microscopic positive surgical margin,
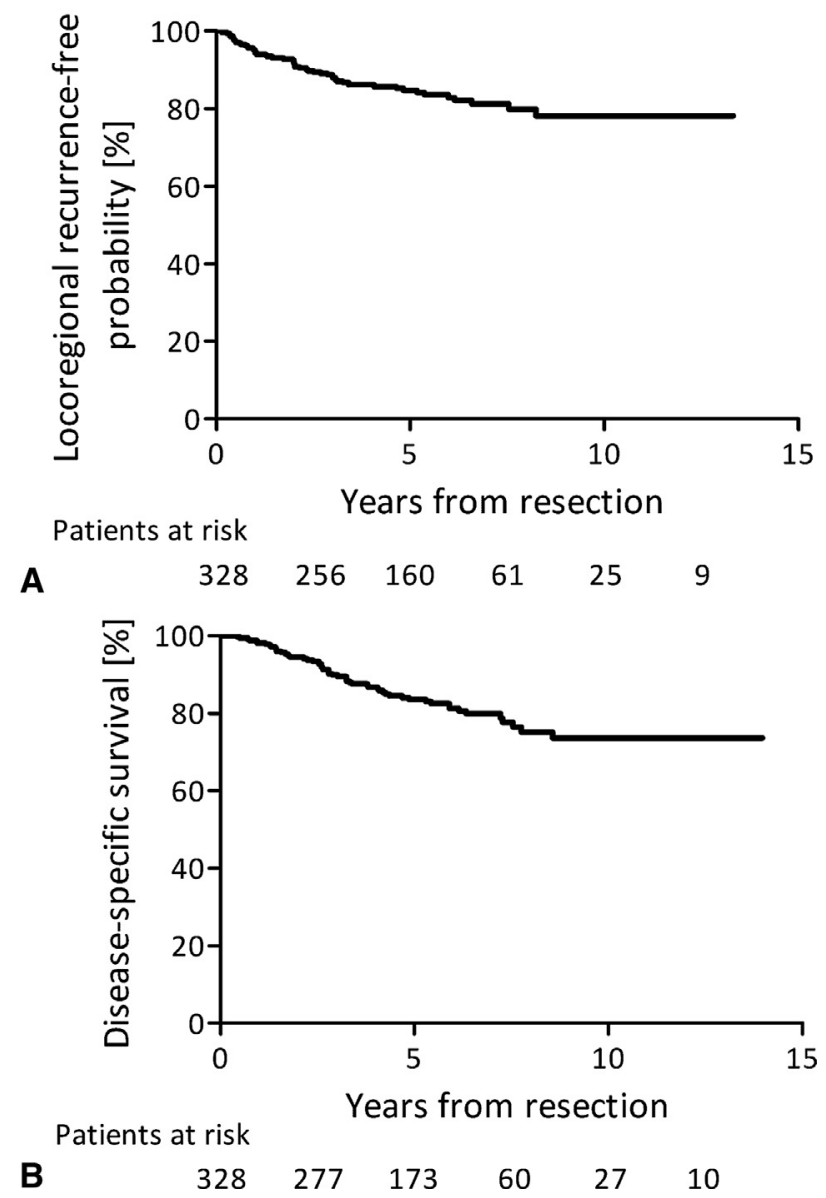

FIGURE 2. Locoregional-free probability (A) and disease-specific survival curves (B) of the 328 patients with clinical stage IA NSCLC undergoing sublobar resection. A, The 5- and 10-year locoregional-free probabilities were $84.8 \%$ and $78.2 \%$, respectively, over a median follow-up period of 58 months. B, The 5- and 10-year disease-specific survivals were $83.6 \%$ and $73.6 \%$, respectively, over a median follow-up period of 62 months. visceral pleural invasion, and lymphatic permeation as independent predictors of locoregional recurrence, and all predictors also were identified as independent predictors of poor disease-specific survival.

Lobectomy has been performed as the standard surgical procedure for operable NSCLC on the basis of the results of a prospective and randomized study of limited resection versus lobectomy conducted by the LCSG. ${ }^{1}$ They reported an increase of the locoregional recurrence rate after limited resection even in patients with small NSCLC $(\leq 3 \mathrm{~cm}$ in size). However, sublobar resection such as segmentectomy and wedge resection could be indicated in patients with NSCLC with comorbid disease or decreased pulmonary function because of its more favorable effect on pulmonary function preservation ${ }^{14}$ and the trend toward reduction in the perioperative morbidity and mortality. ${ }^{15}$ Moreover, various studies of intentional sublobar resection for patients with adequate pulmonary function have been conducted. ${ }^{4,5,7,8}$ In the present study, we sought to investigate patients with clinical stage IA NSCLC who were suitable for sublobar resection who would be identified by the risk factors of locoregional recurrence and poor prognosis.

The extent of pulmonary resection was identified as a significant independent predictor of both locoregional recurrence and poor disease-specific survival. The LCSG reported a trend toward a higher locoregional recurrence rate in patients who underwent wedge resection compared with segmentectomy. ${ }^{1}$ Many studies demonstrated a significantly decreased locoregional recurrence rate and favorable outcomes after segmentectomy compared with wedge resection, ${ }^{6,16}$ and a similar result also was demonstrated even in the study that examined matched comparison groups from a large population-based sample. ${ }^{17}$ Anatomic segmentectomy has the theoretic advantages of providing more comprehensive resection, reduced technical limitations for achieving appropriate margins, and a wider resection of the draining lymphatics, including intersegmental planes, commonly referenced as a source of residual cancer cells. ${ }^{18}$ In the current study, microscopic positive surgical margin on pathologic diagnosis also was found as 
TABLE 2. Multivariate Cox proportional hazard analysis of locoregional recurrence-free probability and disease-specific survival for 328 patients with clinical stage IA non-small cell lung cancer undergoing sublobar resection

\begin{tabular}{|c|c|c|c|c|c|c|}
\hline \multirow[b]{2}{*}{ Variable } & \multicolumn{3}{|c|}{ Locoregional recurrence-free probability } & \multicolumn{3}{|c|}{ Disease-specific survival } \\
\hline & HR & $95 \% \mathrm{CI}$ & $P$ value & HR & $95 \% \mathrm{CI}$ & $P$ value \\
\hline Smoking status $\left(\mathrm{BI}^{*}\right)$ & & & & 1.001 & $1.000-1.001$ & .003 \\
\hline \multicolumn{7}{|c|}{ Pulmonary resection extent } \\
\hline Segmentectomy & Reference & & & Reference & & \\
\hline Wedge resection & 5.787 & $3.018-11.098$ & $<.001$ & 3.183 & $1.756-5.771$ & $<.001$ \\
\hline \multicolumn{7}{|c|}{ Microscopic surgical margin } \\
\hline Positive & 3.888 & $1.634-9.255$ & .002 & 3.211 & $1.427-7.255$ & .005 \\
\hline Negative & Reference & & & Reference & & \\
\hline \multicolumn{7}{|c|}{ Visceral pleural invasion } \\
\hline Absent & Reference & & & Reference & & \\
\hline Present & 2.272 & $1.282-4.027$ & .005 & 2.553 & $1.503-4.338$ & .001 \\
\hline \multicolumn{7}{|l|}{ Lymphatic permeation } \\
\hline Absent & Reference & & & Reference & & \\
\hline Present & 3.824 & $2.028-7.210$ & $<.001$ & 3.223 & $1.773-5.860$ & $<.001$ \\
\hline
\end{tabular}

a significant independent predictor of both locoregional recurrence and poor disease-specific survival. Moreover, the impact of a close surgical margin width on the likelihood of development of local recurrence has been reported, and the authors suggested that the frequent association of wedge resection with a relatively narrower width of the surgical margin was one possible explanation for the higher risk of locoregional recurrence associated with wedge resection. $^{16,19}$ The current study indicates the superior oncologic outcome of segmentectomy compared with wedge resection; thus, segmentectomy should be the surgical procedure of first choice for patients with clinical stage IA NSCLC being considered for sublobar resection.

In the current study, visceral pleural invasion was defined as a risk factor for both locoregional recurrence and poor disease-specific survival. Many previous studies have suggested that visceral pleural invasion is a risk factor for a poor prognosis in patients with NSCLC, ${ }^{20,21}$ and tumors less than $3 \mathrm{~cm}$ in diameter with visceral pleural invasion are upstaged to T2a in the 7th edition of the TNM classification. ${ }^{9}$ Although it is difficult to preoperatively diagnose visceral pleural invasion on CT, measurement of the maximum standardized uptake value of the primary tumor on PET-CT may be a useful marker for predicting pleural invasion. ${ }^{22}$

Lymphovascular space invasion, including vascular and lymphatic invasion, has been reported as one of the independent risk factors for local failure in patients with clinical N0-1 NSCLC undergoing surgical resection. ${ }^{23}$ In addition, we previously reported that the lymphatic permeation is the only independent predictor of both poor prognosis and recurrence in patients with clinical T1a NO NSCLC undergoing intentional segmentectomy. ${ }^{13}$ The present study revealed that lymphatic permeation is a risk factor for locoregional recurrence and poor survival in patients with clinical stage IA NSCLC treated by sublobar resection. However, because we can identify the presence of lymphatic permeation only in the resected lung specimens, we cannot preoperatively take this factor into consideration for determining the indication for sublobar resection. In cases in which lymphatic permeation is identified on pathologic examination of the resected specimen, careful follow-up is recommended.

The patients with a heavy smoking history showed an unfavorable prognosis, although the BI was not identified as an independent predictor for locoregional recurrence. It is generally accepted that cigarette smoking is the strongest risk factor for lung cancer. ${ }^{24}$ In a recent report, existence of an association between a heavy smoking history and poor outcomes with histologic tumor invasion of lung adenocarcinoma was demonstrated, ${ }^{21}$ suggesting that abundant exposure to cigarette smoke affects the incidence rate of lung cancer in smokers, as well as the clinical aggressiveness of the lung cancer.

\section{Study Limitations}

We must acknowledge some limitations of this study. Although the extent of pulmonary resection was identified as a risk factor of locoregional recurrence with a poor prognosis, we did not evaluate the impact of the distance from the margin to the edge of the tumor. Previous studies have shown the impact of a narrow width of the surgical margin on the development of local recurrence; thus, the favorable locoregional control after segmentectomy in the current study might reflect a relatively large width of the surgical margin associated with segmentectomy compared with wedge resection. Moreover, Schuchert and colleagues ${ }^{19}$ suggested that lobectomy should be considered as primary therapy when surgical margins are not obtainable with segmentectomy in low-risk patients. Comparative studies with 
lobectomy, preferably a randomized controlled study, are required to answer this question, best using a randomized study.

Second, it remains controversial whether our postoperative follow-up protocol of chest radiography every 3 months and yearly CT with tumor marker measurements are appropriate for the detection of locoregional recurrence. We considered PET-CT when a pathologic diagnosis could not be obtained despite identification of a newly developed lung lesion or when no recurrence lesion was detected by conventional radiologic modalities, such as $\mathrm{CT}$, brain magnetic resonance imaging, and bone scintigraphy, despite increased tumor markers being identified. PET-CT might be considered as a routine examination to detect locoregional recurrence in asymptomatic patients. ${ }^{25}$

Third, although we evaluated the extent of lymphadenectomy and it was not identified as an independent risk factor for locoregional recurrence, the number of retrieved lymph nodes was not assessed despite these data being potentially as important as the extent of lymphadenectomy. ${ }^{20}$ Moreover, intraoperative lymph node evaluation by frozensection examination was performed only in a limited number of patients; it was rarely performed, especially in high-risk patients for lobectomy, because conversion of the surgical procedure to lobectomy was difficult if positive nodes were identified intraoperatively, and shortening of the operation time was important. These factors could have exerted an influence on the pathologic lymph node status and incidence of intrathoracic lymph node recurrence after resection.

\section{CONCLUSIONS}

Wedge resection, microscopic positive surgical margin, visceral plural invasion, and lymphatic permeation were identified as independent predictors of locoregional recurrence, and all 4 predictors also were identified as independent predictors of poor disease-specific survival in patients with clinical stage IA NSCLC treated by sublobar resection. Segmentectomy may be useful to achieve negative surgical margin with an appropriate width of the margin and should be the surgical procedure of first choice in patients with clinical stage IA NSCLC being considered for sublobar resection. Patients having tumors presenting with no suspicious of pleural involvement would be suitable candidates for sublobar resection. Even after sublobar resection, good local control with a favorable prognosis can be expected in low-risk patients identified by the risk factors in the current study.

\section{References}

1. Ginsberg RJ, Rubinstein LV. Randomized trial of lobectomy versus limited resection for T1 N0 non-small cell lung cancer. Lung Cancer Study Group. Ann Thorac Surg. 1995;60:615-23.

2. Ginsberg RJ. Limited resection in the treatment of stage I non-small cell lung cancer; an overview. Chest. 1989;96(1 Suppl):50S-1S.
3. Kilic A, Schuchert MJ, Pettiford BL, Pennathur A, Landreneau JR, Landreneau JP, et al. Anatomic segmentectomy for stage I non-small cell lung cancer in the elderly. Ann Thorac Surg. 2009;87:1662-8.

4. Koike T, Yamato Y, Yoshiya K, Shimoyama T, Suzuki R. Intentional limited pulmonary resection for peripheral T1 N0 M0 small-sized lung cancer. J Thorac Cardiovasc Surg. 2003;125:924-8.

5. Okada M, Koike T, Higashiyama M, Yamato Y, Kodama K, Tsubota N. Radical sublobar resection for small-sized non-small cell lung cancer: a multicenter study. J Thorac Cardiovasc Surg. 2006;132:769-75.

6. Okada M, Nishio W, Sakamoto T, Uchino K, Yuki T, Nakagawa A, et al. Effect of tumor size on prognosis in patients with non-small cell lung cancer: the role of segmentectomy as a type of lesser resection. J Thorac Cardiovasc Surg. 2005; 129:87-93.

7. Yoshida J, Nagai K, Yokose T, Nishimura M, Kakinuma R, Ohmatsu H, et al. Limited resection trial for pulmonary ground-glass opacity nodules: fifty-case experience. J Thorac Cardiovasc Surg. 2005;129:991-6.

8. Koike T, Togashi K, Shirato T, Sato S, Hirahara M, Sugawara M, et al. Limited resection for noninvasive bronchioloalveolar carcinoma diagnosed by intraoperative pathologic examination. Ann Thorac Surg. 2009;88:1106-11.

9. Goldstraw P, Crowley J, Chansky K, Giroux DJ, Groome PA, Rami-Porta R, et al. The IASLC Lung Cancer Staging Project: proposals for the revision of the TNM stage groupings in the forthcoming (seventh) edition of the TNM Classification of malignant tumours. J Thorac Oncol. 2007;2:706-14.

10. Suzuki K, Koike T, Asakawa T, Kusumoto M, Asamura H, Nagai K, et al. A prospective radiological study of thin-section computed tomography to predict pathological noninvasiveness in peripheral clinical IA lung cancer (Japan Clinical Oncology Group 0201). J Thorac Oncol. 2011;6:751-6.

11. Brinkman GL, Coates EO Jr. The effect of bronchitis, smoking, and occupation on ventilation. Am Rev Respir Dis. 1963;87:684-93.

12. Kondo K, Takahashi Y, Hirose Y, Nagao T, Tsuyuguchi M, Hashimoto M, et al. The reduced expression and aberrant methylation of p16(INK4a) in chromate workers with lung cancer. Lung Cancer. 2006;53:295-302.

13. Koike T, Koike T, Yamato Y, Yoshiya K, Toyabe S. Prognostic predictors in nonsmall cell lung cancer patients undergoing intentional segmentectomy. Ann Thorac Surg. 2012;93:1788-94

14. Harada H, Okada M, Sakamoto T, Matsuoka H, Tsubota N. Functional advantage after radical segmentectomy versus lobectomy for lung cancer. Ann Thorac Surg. 2005;80:2041-5.

15. Schuchert MJ, Kilic A, Pennathur A, Nason KS, Wilson DO, Luketich JD, et al. Oncologic outcomes after surgical resection of subcentimeter non-small cell lung cancer. Ann Thorac Surg. 2011;91:1681-8.

16. El-Sherif A, Fernando HC, Santos R, Pettiford B, Luketich JD, Close JM, et al. Margin and local recurrence after sublobar resection of non-small cell lung cancer. Ann Surg Oncol. 2007;14:2400-5.

17. Smith CB, Swanson SJ, Mhango G, Wisnivesky JP. Survival after segmentectomy and wedge resection in stage I non-small-cell lung cancer. J Thorac Oncol. 2013;8:73-8.

18. Blasberg JD, Pass HI, Donington JS. Sublobar resection: a movement from the Lung Cancer Study Group. J Thorac Oncol. 2010;5:1583-93.

19. Schuchert MJ, Pettiford BL, Keeley S, D'Amato TA, Kilic A, Close J, et al. Anatomic segmentectomy in the treatment of stage I non-small cell lung cancer. Ann Thorac Surg. 2007;84:926-33.

20. Hsu CP, Hsia JY, Chang GC, Chuang CY, Shai SE, Yang SS, et al. Surgical-pathologic factors affect long-term outcomes in stage IB (pT2 N0 M0) non-small cell lung cancer: a heterogeneous disease. J Thorac Cardiovasc Surg. 2009;138:426-33.

21. Maeda R, Yoshida J, Ishii G, Hishida T, Nishimura M, Nagai K. Influence of cigarette smoking on survival and tumor invasiveness in clinical stage IA lung adenocarcinoma. Ann Thorac Surg. 2012;93:1626-32.

22. Al-Sarraf N, Gately K, Lucey J, Aziz R, Doddakula K, Wilson L, et al. Clinical implication and prognostic significance of standardised uptake value of primary non-small cell lung cancer on positron emission tomography: analysis of 176 cases. Eur J Cardiothorac Surg. 2008;34:892-7.

23. Kelsey CR, Marks LB, Hollis D, Hubbs JL, Ready NE, D'Amico TA, et al. Local recurrence after surgery for early stage lung cancer: an 11-year experience with 975 patients. Cancer. 2009;115:5218-27.

24. Parkin DM, Pisani P, Lopez AD, Masuyer E. At least one in seven cases of cancer is caused by smoking. Global estimates for 1985. Int J Cancer. 1994;59:494-504.

25. Toba H, Sakiyama S, Otsuka H, Kawakami Y, Takizawa H, Kenzaki K, et al. $18 \mathrm{~F}$-fluorodeoxyglucose positron emission tomography/computed tomography is useful in postoperative follow-up of asymptomatic non-small-cell lung cancer patients. Interact Cardiovasc Thorac Surg. 2012;15:859-64. 